\title{
Closed-Form Barrier Functions for Multi-Agent Ellipsoidal Systems with Uncertain Lagrangian Dynamics
}

\author{
Christos K. Verginis and Dimos V. Dimarogonas
}

\begin{abstract}
In this paper, we design a decentralized control protocol for the collision avoidance of a multi-agent system, which is comprised of $3 \mathrm{D}$ ellipsoidal agents that obey 2 nd-order uncertain Lagrangian dynamics. More specifically, we derive a novel closed-form smooth barrier function that resembles a distance metric between $3 \mathrm{D}$ ellipsoids and can be used by feedback-based control laws to guarantee inter-agent collision avoidance. Discontinuities and adaptation laws are incorporated in the control protocol to deal with the uncertainties of the dynamic model. The control laws are decentralized, in the sense that each agent uses only local sensing information. Simulation results verify the theoretical findings.
\end{abstract}

Index Terms-Cooperative control, Decentralized control, Agents-based systems, Robust adaptive control

\section{INTRODUCTION}

C OLLISION avoidance in systems comprised of multiple robotic agents is a crucial safety property that needs to be always achieved. Except for the single-agent case [1], [2], multi-agent collision avoidance is tackled in a variety of works (e.g., [3]-[8]), where the multi-agent system aims for a primary objective (navigation, formation). The majority of the related works considers spherical agents, which provide a straightforward metric for the inter-agent or the agent-toobstacle distances. However, since the shapes of real robotic vehicles can be far from spherical (e.g., robotic manipulators), that approach can be too conservative and may prevent the agents from fulfilling their primary objectives. Ellipsoids, on the other hand, can approximate more accurately the volume of autonomous agents (see Fig. 1).

The authors in [1], [9], [10] employ diffeomorphisms to transform arbitrarily-shaped obstacles, including ellipsoids, to points. This methodology, however, is not straightforwardly extendable to the case of moving obstacles (i.e., multiple autonomous agents). A point-world transformation of multiagent systems was taken into account in [11], [12]. As described in [11] though, each agent's transformation deforms the other agents into shapes whose implicit closed-form equation (and hence a suitable distance metric) is not trivial to obtain. The methodology of [9] provides useful insight, where

The authors are with the KTH Center of Autonomous Systems, School of Electrical Engineering and Computer Science, KTH Royal Institute of Technology, SE-100 44, Stockholm, Sweden. Email: \{cverginis, dimos $\}$ akth. se. This work was supported by the H2020 ERC Starting Grant BUCOPHSYS, the European Union's Horizon 2020 Research and Innovation Programme under the GA No. 731869 (Co4Robots), the Swedish Research Council (VR), the Knut och Alice Wallenberg Foundation (KAW) and the Swedish Foundation for Strategic Research (SSF). the volume of each agent is "absorbed" to the other agents via Minkowski sums. The closed-form implicit equation of the resulting shapes, however, although possible to obtain [13], cannot be used to derive an appropriate distance metric in a straightforward way; [14] derives a conservative inter-ellipsoid distance by employing ellipsoid-to-sphere transformations and eigenvalue computations. An arithmetic algorithm that produces velocities for inter-agent elliptical agents is derived in [15], without, however, theoretical guarantees. Optimizationbased techniques (e.g., Model Predictive Control), which can be employed for collision avoidance of convex-shaped agents [16], can be too complex to solve, especially in cases where the control must be decentralized and/or complex dynamics are considered. The latter property constitutes another important issue regarding the related literature. In particular, most related works consider simplified single- or double-integrator models, which deviate from the actual dynamics and can lead to performance decline and safety jeopardy.

Barrier functions constitute a suitable tool for expressing objectives like collision avoidance. Originated in optimization, they are continuous functions that diverge to infinity as their argument approaches the boundary of a desired/feasibly region. Barrier Lyapunov-like functions for general control systems can be found in [17], [18], and in [3], [19], [20] for multi-agent systems, for obstacle avoidance with spherical obstacles/agents and time-dependent tasks.

According to the authors's best knowledge, there are no existing works addressing collision avoidance between 3D ellipsoidal agents subject to dynamic uncertainties and external disturbances under closed-form control protocols, which is the focus of this work. In particular, we design smooth closedform barrier functions for the collision avoidance of ellipsoidal agents. By employing results from the computer graphics field, we derive a novel closed-form expression that represents a distance metric ${ }^{1}$ of two ellipsoids in 3D space. Moreover, we use the latter to design a control protocol that guarantees the collision avoidance of a multi-agent system that aims to achieve a primary objective, subject to uncertain 2nd-order Lagrangian dynamics. The derived control law is (i) decentralized, in the sense that each agent calculates its control signal based on local information, (ii) discontinuous and adaptive, in order to compensate for the uncertainties and external disturbances. We note that the derived barrier functions have

\footnotetext{
${ }^{1}$ By distance metric we mean that it is zero in a collision between the ellipsoids and positive otherwise.
} 
appeared in our preliminary results [21], incorporated however with simpler dynamics. This work provides significant improvements, also from a practical viewpoint, by considering external disturbances and more general uncertainties in the agents' dynamics. Moreover, in contrast to [21], we present here an important symmetric property of the derived barrier functions (Proposition 3) that plays a key role in the multiagent control design procedure.

The rest of the paper is organized as follows. Section II provides preliminary background and the used notation. Section III formulates the treated problem and Section IV illustrates the main results. Section $V$ is devoted to a simulation example and Section VI concludes the paper.
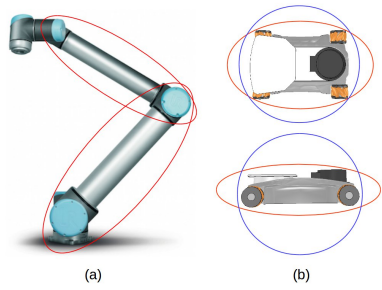

Fig. 1. Ellipsoid approximation of (a) the rigid links of a robotic manipulator, (b) a mobile robot (top and front view).

\section{NOTATION AND PRELIMINARIES}

\section{A. Notation}

The sets of natural and real numbers are denoted by $\mathbb{N}$, and $\mathbb{R}$, respectively, and $\mathbb{R}_{>0}, \mathbb{R}_{>0}$ are the sets of nonnegative and positive real numbers, respectively; $\|x\|_{1}$ and $\|x\|$ denote the 1- and 2-norm, respectively, of a vector $x \in \mathbb{R}^{n} ; \mathrm{SE}(3)$ is the special Euclidean group and $\mathrm{S}^{n-1}$ is the $n$-dimensional sphere. Given a set $A$, its interior is denoted by $\AA$. The identity matrix is $I_{n} \in \mathbb{R}^{n \times n}$. The open and closed balls with radius $\delta$, centered at $x \in \mathbb{R}^{n}$, are denoted by $\mathcal{B}(x, \delta)$ and $\overline{\mathcal{B}}(x, \delta)$, respectively. The sign function is defined as $\operatorname{sgn}(x)=-1$, if $x<0$, $\operatorname{sgn}(x)=0$, if $x=0$, and $\operatorname{sgn}(x)=1$, if $x>0$; its vector counterpart is defined as $\operatorname{sgn}(x)=\left[\operatorname{sgn}\left(x_{1}\right), \ldots, \operatorname{sgn}\left(x_{n}\right)\right]^{\top} \in$ $\mathbb{R}^{n}$, for $x=\left[x_{1}, \ldots, x_{n}\right]^{\top} \in \mathbb{R}^{n}$. Given a discontinuous function $f: \mathbb{R}^{n} \rightarrow \mathbb{R}^{k}$, its Filippov regularization [22] is defined as $\mathrm{K}[f](x):=\bigcap_{\delta>0} \bigcap_{\mu(\bar{N})=0} \overline{\operatorname{co}}(f(\mathcal{B}(x, \delta) \backslash \bar{N}), t)$, where $\bigcap_{\mu(\bar{N})=0}$ is the intersection over all sets $\bar{N}$ of Lebesgue measure zero, and $\overline{\mathrm{co}}(E)$ is the convex closure of the set $E$. The Filippov regularization of $\operatorname{sgn}(x) \in \mathbb{R}$ is denoted by $\mathrm{K}[\operatorname{sgn}](x)=\operatorname{SGN}(x)$ where $\operatorname{SGN}(x):=-1$, if $x<0$, $\operatorname{SGN}(x):=1$, if $x>0$, and $\operatorname{SGN}(x) \in[-1,1]$, if $x=0$.

\section{B. Cubic Equations and Ellipsoid Collision}

Proposition 1: Consider the cubic equation $f(\lambda)=c_{3} \lambda^{3}+$ $c_{2} \lambda^{2}+c_{1} \lambda+c_{0}=0$ with $c_{\ell} \in \mathbb{R}, \forall \ell \in\{0, \ldots, 3\}$ and roots $\lambda_{1}, \lambda_{2}, \lambda_{3}$, with $f\left(\lambda_{1}\right)=f\left(\lambda_{2}\right)=f\left(\lambda_{3}\right)=0$. Then, given its discriminant $\Delta:=\left(c_{3}\right)^{4} \prod_{\substack{i \in\{1,2\} \\ j \in\{i+1, \ldots, 3\}}}\left(\lambda_{i}-\lambda_{j}\right)^{2}$, the following hold:

(i) $\Delta=0 \Leftrightarrow \exists i, j \in\{1,2,3\}$, with $i \neq j$, such that $\lambda_{i}=$ $\lambda_{j}$, i.e., at least two roots are equal, (ii) $\Delta>0 \Leftrightarrow \lambda_{i} \in \mathbb{R}, \forall i \in\{1,2,3\}$, and $\lambda_{i} \neq \lambda_{j}, \forall i, j \in$ $\{1,2,3\}$, with $i \neq j$, i.e., all roots are real and distinct.

Proposition 2: [23] Consider two planar ellipsoids $\mathcal{A}=$ $\left\{z \in \mathbb{R}^{3}\right.$ s.t. $\left.z^{\top} A(t) z \leq 0\right\}, \mathcal{B}=\left\{z \in \mathbb{R}^{3}\right.$ s.t. $\left.z^{\top} B(t) z \leq 0\right\}$, with $z=\left[p^{\top} 1\right]^{\top}, p \in \mathbb{R}^{2}$, and $A, B: \mathbb{R}_{\geq 0} \rightarrow \mathbb{R}^{3 \times 3}$ terms that describe their motion in 2D space. Given their characteristic polynomial $f(\lambda)=\operatorname{det}(\lambda A-B)$, which has degree 3 , the following hold:

(i) $\exists \lambda^{*}>0$ s.t. $f\left(\lambda^{*}\right)=0$, i.e, the polynomial $f(\lambda)$ has always one positive real root,

(ii) $\mathcal{A} \cap \mathcal{B}=\emptyset$ if and only if the characteristic equation $f(\lambda)=0$ has two distinct negative roots, i.e., $\exists \lambda_{1}^{*}, \lambda_{2}^{*}<$ 0 , with $\lambda_{1}^{*} \neq \lambda_{2}^{*}$, and $f\left(\lambda_{1}^{*}\right)=f\left(\lambda_{2}^{*}\right)=0$.

(iii) $\mathcal{A} \cap \mathcal{B} \neq \emptyset$ and $\mathcal{A} \cap \mathcal{B}=\emptyset$, i.e., $\mathcal{A}$ and $\mathcal{B}$ touch externally, if and only if and only if $f(\lambda)=0$ has a negative root with multiplicity 2 .

\section{Nonsmooth Analysis}

Consider the following differential equation with a discontinuous right-hand side:

$$
\dot{x}=f(x, t),
$$

where $f: \mathcal{D} \times\left[t_{0}, \infty\right) \rightarrow \mathbb{R}^{n}, \mathcal{D} \subset \mathbb{R}^{n}$, is Lebesgue measurable and locally essentially bounded.

Definition 1 (Def. 1 of [24]): A function $x:\left[t_{0}, t_{1}\right) \rightarrow \mathbb{R}^{n}$, with $t_{1}>t_{0}$, is called a Filippov solution of (1) on $\left[t_{0}, t_{1}\right)$ if $x(t)$ is absolutely continuous and if, for almost all $t \in\left[t_{0}, t_{1}\right)$, it satisfies $\dot{x} \in \mathrm{K}[f](x, t)$.

Lemma 1 (Lemma 1 of [24]): Let $x(t)$ be a Filippov solution of (1) and $V: \mathcal{D} \times\left[t_{0}, t_{1}\right) \rightarrow \mathbb{R}$ be a locally Lipschitz, regular function ${ }^{2}$. Then $V(x(t), t)$ is absolutely continuous, $\frac{\partial}{\partial t} V(x(t), t)$ exists almost everywhere (a.e.), i.e., for almost all $t \in\left[t_{0}, t_{1}\right)$, and $\dot{V}(x(t), t) \stackrel{\text { a.e }}{\in} \dot{\widetilde{V}}(x(t), t):=$ $\cap_{\xi \in \partial V(x, t)} \xi^{\top}\left[\mathrm{K}[f](x, t)^{\top}, 1\right]^{\top}$, where $\partial V(x, t)$ is the Clarke's generalized gradient [24].

Theorem 1 (Corollary 2 of [24]): For the system given in (1), let $\mathcal{D} \subset \mathbb{R}^{n}$ be an open and connected set containing $x=0$ and suppose that $f$ is Lebesgue measurable and $x \mapsto$ $f(x, t)$ is essentially locally bounded, uniformly in $t$. Let $V$ : $\mathcal{D} \times\left[t_{0}, t_{1}\right) \rightarrow \mathbb{R}$ be locally Lipschitz and regular such that $W_{1}(x) \leq V(x, t) \leq W_{2}(x), \forall t \in\left[t_{0}, t_{1}\right), x \in \mathcal{D}$, and $z \leq$ $-W(x(t)), \quad \forall z \in \dot{\tilde{V}}(x(t), t), t \in\left[t_{0}, t_{1}\right), x \in \mathcal{D}$, where $W_{1}$ and $W_{2}$ are continuous positive definite functions and $W$ is a continuous positive semi-definite on $\mathcal{D}$. Choose $r>0$ and $c>0$ such that $\overline{\mathcal{B}}(0, r) \subset \mathcal{D}$ and $c<\min _{\|x\|=r} W_{1}(x)$. Then for all Filippov solutions $x:\left[t_{0}, t_{1}\right) \rightarrow \mathbb{R}^{n}$ of (1), with $x\left(t_{0}\right) \in \overline{\mathcal{D}}:=\left\{x \in \overline{\mathcal{B}}(0, r): W_{2}(x) \leq c\right\}$, it holds that $t_{1}=\infty, x(t) \in \overline{\mathcal{D}}, \forall t \in\left[t_{0}, \infty\right)$, and $\lim _{t \rightarrow \infty} W(x(t))=0$.

\section{PROBLEM FORMULATION}

Consider $N>1$ ellipsoidal autonomous agents, with $\mathcal{N}:=\{1, \ldots, N\}$, operating in $\mathrm{SE}(3)$, and described by the ellipsoids $\mathcal{A}_{i}\left(x_{i}\right):=\left\{y \in \mathbb{R}^{4}: y^{\top} A_{i}\left(x_{i}\right) y \leq 0\right\}$; $x_{i}:=\left[p_{i}^{\top}, \eta_{i}^{\top}\right]^{\top} \in \mathbb{M}:=\mathbb{R}^{3} \times \mathrm{S}^{3}$ is the $i$ th agent's center of mass pose, where $p_{i} \in \mathbb{R}^{3}$ is its inertial position and

\footnotetext{
${ }^{2}$ See [24] for a definition of regular functions.
} 
$\eta_{i}:=\left[\varphi_{i}, \epsilon_{i}^{\top}\right]^{\top} \in S^{3}$ its unit quaternion-based orientation,with $\varphi_{i} \in \mathbb{R}, \epsilon_{i} \in \mathbb{R}^{3}$ its scalar and vector parts, respectively, subject to $\left\|\eta_{i}\right\|=1 ; A_{i}\left(x_{i}\right):=T_{i}^{-\top}\left(x_{i}\right) \hat{A}_{i} T_{i}^{-1}\left(x_{i}\right)$, with $\hat{A}_{i}:=\operatorname{diag}\left\{l_{x, i}^{-2}, l_{y, i}^{-2}, l_{z, i}^{-2},-1\right\}$, corresponding to the principal axis lengths $l_{x, i}, l_{y, i}, l_{z, i} \in \mathbb{R}_{>0}$ of agent $i$ 's ellipsoid, and $T_{i} \in \mathrm{SE}(3)$ is the transformation matrix describing the translation and orientation of agent $i$ 's center of mass, $\forall i \in \mathcal{N}$. The agents' motion follows the 2nd-order dynamics:

$$
\begin{aligned}
& \dot{x}_{i}=\bar{E}_{\eta}\left(\eta_{i}\right) v_{i} \\
& M_{i}\left(x_{i}\right) \dot{v}_{i}+C_{i}\left(x_{i}, v_{i}\right) v_{i}+g_{i}\left(x_{i}\right)+f_{i}\left(v_{i}\right)+d_{i}(t)=u_{i},
\end{aligned}
$$

where $v_{i}:=\left[\dot{p}_{i}^{\top}, \omega_{i}^{\top}\right]^{\top}$ is agent $i$ 's velocity, with $\omega_{i} \in \mathbb{R}^{3}$ being its angular velocity, $\bar{E}_{\eta}: S^{3} \rightarrow \mathbb{R}^{7 \times 6}$ is the matrix mapping the quaternion rates to velocities [25], $M_{i}: \mathbb{M} \rightarrow \mathbb{R}^{6 \times 6}$ are positive definite inertia matrices, satisfying the property $\underline{m} \leq M_{i}(x) \leq \bar{m}, \forall x \in \mathbb{M}, i \in \mathcal{N}$, for positive constants $\underline{m}, \bar{m}, C_{i}: \mathbb{M} \times \mathbb{R}^{6} \rightarrow \mathbb{R}^{n \times n}$ are the Coriolis terms, $g_{i}: \mathbb{M} \rightarrow \mathbb{R}^{6}$ are the gravity vectors, $f_{i}: \mathbb{R}^{6} \rightarrow \mathbb{R}^{6}$ are unknown vector fields that represent static friction-like terms, $d_{i}: \mathbb{R}_{\geq 0} \rightarrow \mathbb{R}^{6}$ are unknown external disturbances, and $u_{i} \in \mathbb{R}^{\overline{6}}$ are the robots' control inputs, $\forall i \in \mathcal{N}$. The terms $M_{i}, C_{i}$ and $g_{i}$ are continuous everywhere, the terms $f_{i}$ are locally bounded and continuous almost everywhere, and $d_{i}$ are measurable and uniformly bounded. We also consider that $u_{i}$ is decomposed as $u_{i}=u_{f, i}+u_{s, i}$, where $u_{f_{i}}$ is a bounded term that is responsible for some (potentially cooperative) task, and $u_{s, i}$ is a control term to be designed in order to achieve multi-agent decentralized collision avoidance, $\forall i \in \mathcal{N}$. More specifically, we consider that $\phi(x) \in \mathbb{R}_{\geq 0}$ is a term that corresponds to the cooperative task dictated by $u_{f, i}$, with $u_{f, i}=\bar{E}_{\eta}\left(\eta_{i}\right)^{\top} \frac{\partial \phi(x)}{\partial x_{i}}, \forall i \in \mathcal{N}, c_{1}(x) \leq \phi(x) \leq$ $c_{2}(x)$, for continuous positive definite functions $c_{1}, c_{2}$, and nonempty sets $\left\{x \in \mathcal{X}: x=\phi^{-1}(y)\right\}, \forall y \in \mathbb{R}_{\geq 0}$, where $x:=\left[x_{1}^{\top}, \ldots, x_{N}^{\top}\right]^{\top}$, and $\mathcal{X}:=\left\{x \in \mathbb{M}^{N}: \mathcal{A}_{i}\left(x_{i}\right) \cap\right.$ $\left.\mathcal{A}_{j}\left(x_{j}\right)=\emptyset, \forall i, j \in \mathcal{N}, i \neq j\right\} ; \phi$ can be also a function of $\widetilde{x}:=\left[p_{1}^{\top}-p_{2}^{\top}, \ldots, p_{N}^{\top}-p_{N-1}^{\top}, \eta_{1}^{\top}, \ldots, \eta_{N}^{\top}\right]^{\top}$ that concerns potential formation control objectives. Then $\mathcal{X}$ becomes $\{\widetilde{x} \in$ $\left.\mathbb{R}^{\frac{3 N(N-1)}{2}} \times \mathrm{S}^{3 N}: \mathcal{A}_{i}\left(x_{i}\right) \cap \mathcal{A}_{j}\left(x_{j}\right)=\emptyset, \forall i, j \in \mathcal{N}, i \neq j\right\}$. The conditions for $\phi$ are satisfied by standard quadratic functions, e.g., $\phi(x)=\sum_{i \in \mathcal{N}}\left\{\left\|p_{i}-\alpha_{i}\right\|^{2}+e_{\eta_{i}}^{2}\right\}$ (for multi-agent navigation) or $\phi(\widetilde{x})=\sum_{(i, j) \in \mathcal{F}}\left\{\left\|p_{i}-p_{j}-\alpha_{i, j}\right\|^{2}+e_{\eta_{i, j}}^{2}\right\}$ (for formation) for sufficiently distant $\alpha_{i}, \alpha_{i, j}$, where $\mathcal{F}$ is a potential formation set and $e_{\eta_{i}}, e_{\eta_{i, j}}$ represent appropriate quaternion errors [25]. Note that $\phi$ and $u_{f, i}$ are not responsible for collision avoidance or compensating model uncertainties.

The dynamics (2) have the following properties [26]:

Property 1: The terms $C_{i}$ can be chosen such that $\dot{M}_{i}(x)-2 C_{i}(x, \dot{x})$ are skew-symmetric, i.e., $y^{\top}\left(\dot{M}_{i}(x)-\right.$ $\left.2 C_{i}(x, \dot{x})\right) y=0, \forall x \in \mathbb{M}, \dot{x}, y \in \mathbb{R}^{6}, i \in \mathcal{N}$.

Property 2: The gravity terms of (2) can be written as $g_{i}(x)=Y_{i}(x) \theta_{i}, \forall x \in \mathbb{M}, i \in \mathcal{N}$, where $Y_{i}: \mathbb{M} \rightarrow \mathbb{R}^{6 \times \ell}$ are known continuous matrices, and $\theta_{i} \in \mathbb{R}^{\ell}, \ell \in \mathbb{N}$, are constant but unknown dynamic parameters of the agents, $\forall i \in \mathcal{N}$.

Property 3: [26] The friction terms are dissipative, i.e., $v_{i}^{\top} f_{i}\left(v_{i}\right)>0, \forall v_{i} \neq 0, i \in \mathcal{N}$.

Moreover, the following assumption is needed:

Assumption 1: It holds that $\left\|d_{i}(t)\right\|_{1} \leq d_{b_{i}}, \forall t \in \mathbb{R}_{\geq 0}$, where $d_{b_{i}}$ are unknown positive constants, $i \in \mathcal{N}$.
Note that in our previous work [21] we imposed a growth condition on the terms $f_{i}(\cdot)$ and we did not consider any form of external disturbances. In addition, we consider that each robot has a limited sensing radius $d_{\mathrm{con}, i} \in \mathbb{R}_{>0}$, with $d_{\mathrm{con}, i}>$ $\max \left\{l_{x, i}, l_{y, i}, l_{z, i}\right\}+\max _{j \in \mathcal{N}}\left\{\max \left\{l_{x, j}, l_{y, j}, l_{z, j}\right\}\right\}+\varepsilon$ for an arbitrarily small positive constant $\varepsilon$, which implies that the agents can sense each other without colliding. Based on this, we can model the topology of the multi-robot network through the undirected time-varying graph $\mathcal{G}(p):=(\mathcal{N}, \mathcal{E}(p))$, with $\mathcal{E}(p):=\left\{(i, j) \in \mathcal{N}^{2}:\left\|p_{i}-p_{j}\right\| \leq \min \left\{d_{\text {con }, i}, d_{\text {con }, j}\right\}\right\}, p:=$ $\left[p_{1}^{\top}, \ldots, p_{N}^{\top}\right]^{\top}$, and we further define the agent time-varying neighborhood $\mathcal{N}_{i}(p):=\left\{j \in \mathcal{N}:\left\|p_{i}-p_{j}\right\|<d_{\text {con }, i}\right\}, \forall i \in \mathcal{N}$. Moreover, we consider the complete graph $\overline{\mathcal{G}}:=(\mathcal{N}, \overline{\mathcal{E}})$, with $\overline{\mathcal{E}}:=\{(i, j), \forall i, j \in \mathcal{N}, i<j\}, \bar{M}:=|\overline{\mathcal{E}}|=\frac{N(N-1)}{2}$ and an edge numbering set $\overline{\mathcal{M}}:=\{1, \ldots, \bar{M}\}$. Finally, given an edge $m \in \overline{\mathcal{M}}$, we use the notation $\left(m_{1}, m_{2}\right) \in \mathcal{N}^{2}$ for the robot indices of edge $m \in \overline{\mathcal{M}}$. As discussed in Section I, the agents need to avoid collisions with each other, while executing their task, dictated by $u_{f, i}$. To that end, we aim to design closedform barrier functions and decentralized feedback control laws $u_{s, i}$ that guarantee collision avoidance among the ellipsoidal agents, while compensating appropriately for the model uncertainties and the external disturbances. By "decentralized", we mean here that agents can use only local information, in view of their limited sensing radius (i.e., only $p_{j}, \eta_{j}, j \in \mathcal{N}_{i}(p)$ ). Formally, the treated problem is the following:

Problem 1: Given $N$ 3D ellipsoidal autonomous agents with the uncertain Lagrangian dynamics (2) executing tasks dictated by $u_{f, i}$, design

1) closed-form barrier functions that encode collision avoidance of the agents,

2) decentralized control laws in $u_{s, i}$ that guarantee interagent collision avoidance, i.e., $\mathcal{A}_{i}\left(x_{i}(t)\right) \cap \mathcal{A}_{j}\left(x_{j}(t)\right)=\emptyset$, $\forall i, j \in \mathcal{N}, i \neq j$, as well as boundedness of all closed loop signals.

\section{MAIN RESULTS}

This section describes the proposed solution to Problem 1. In order to deal with the ellipsoidal collision avoidance, we employ results from computer graphics that are related to detection of ellipsoid collision and we build appropriate barrier functions whose boundedness implies the collisionfree trajectories. Moreover, we use adaptive and discontinuous control laws to appropriately compensate for the uncertainties and external disturbances of (2).

We employ first the results described in Proposition 2 to build an appropriate ellipsoidal barrier function. Note, however, that these results concern planar ellipsoids and cannot be straightforwardly extended to the 3D case, which is the case of the considered multi-agent system. For that reason, we consider the respective planar projections. For an ellipsoid $\mathcal{A}_{i}, i \in \mathcal{N}$, we denote as $\mathcal{A}_{i}^{x y}, \mathcal{A}_{i}^{x z}, \mathcal{A}_{i}^{y z}$ its projections on the planes $x-y, x-z$ and $y$-z, respectively, with corresponding matrix terms $A_{i}^{x y}, A_{i}^{x z}, A_{i}^{y z}$ (i.e., $\mathcal{A}_{i}^{s}\left(x_{i}\right):=$ $\left.\left\{y \in \mathbb{R}^{3}: y^{\top} A_{i}^{s}\left(x_{i}\right) y \leq 0\right\}, \forall s \in\{x y, x z, y z\}\right)$. Note that in order for $\mathcal{A}_{i}, \mathcal{A}_{j}$ to collide (touch externally), all their projections on the three planes must also collide, i.e., 
$\mathcal{A}_{i}\left(x_{i}\right) \cap \mathcal{A}_{j}\left(x_{j}\right) \neq \emptyset \wedge \stackrel{\circ}{\mathcal{A}}_{i}\left(x_{i}\right) \cap \stackrel{\mathcal{A}}{j}_{j}\left(x_{j}\right)=\emptyset \Leftrightarrow \mathcal{A}_{i}^{s}\left(x_{i}\right) \cap$ $\mathcal{A}_{j}^{s}\left(x_{j}\right) \neq \emptyset \wedge \stackrel{\circ}{\mathcal{A}}_{i}^{s}\left(x_{i}\right) \cap \stackrel{\mathcal{A}}{j}_{j}^{s}\left(x_{j}\right)=\emptyset, \forall s \in\{x y, x z, y z\}$, i.e., Therefore, $\mathcal{A}_{i}$ and $\mathcal{A}_{j}$ do not collide if and only if $\mathcal{A}_{i}^{s}\left(x_{i}\right) \cap \mathcal{A}_{j}^{s}\left(x_{j}\right)=\emptyset$ for some $s \in\{x y, x z, y z\}$. In view of Proposition 2, that means that the characteristic equations $f_{i, j}^{s}(\lambda):=\operatorname{det}\left(\lambda A_{i}^{s}\left(x_{i}\right)-A_{j}^{s}\left(x_{j}\right)\right)=0$ must always have one positive real root and two negative distinct roots for at least one $s \in\{x y, x z, y z\}$. Hence, by denoting the discriminant of $f_{i, j}^{s}(\lambda)=0$ as $\Delta_{i, j}^{s}\left(x_{i}, x_{j}\right)$, Proposition 1 suggests that $\Delta_{i, j}^{s}\left(x_{i}, x_{j}\right)$ must remain always positive for at least one $s \in\{x y, x z, y z\}$, since a collision would imply $\Delta_{i, j}^{s}\left(x_{i}, x_{j}\right)=$ $0, \forall s \in\{x y, x z, y z\}$. Therefore, by defining the smooth function [9] $\sigma(x):=\exp \left(-\frac{1}{x}\right)$, if $x>0$ and $\sigma(x):=0$, if $x \leq 0$, we conclude that $\mathcal{A}_{i}$ and $\mathcal{A}_{j}$ do not collide if and only if $\sigma\left(\Delta_{i, j}^{x y}\left(x_{i}, x_{j}\right)\right)+\sigma\left(\Delta_{i, j}^{x z}\left(x_{i}, x_{j}\right)\right)+\sigma\left(\Delta_{i, j}^{y z}\left(x_{i}, x_{j}\right)\right)>$ 0 , since a collision would result in $\Delta_{i, j}^{s}\left(x_{i}, x_{j}\right)=0 \Leftrightarrow$ $\sigma\left(\Delta_{i, j}^{s}\left(x_{i}, x_{j}\right)\right)=0, \forall s \in\{x y, x z, y z\}$. We aim now at defining a decentralized continuously differentiable function for each edge $m \in \overline{\mathcal{M}}$ that incorporates the collision avoidance property of agents $m_{1}, m_{2}$. We need first the following result regarding the discriminant of $f_{i, j}^{s}(\lambda)=0$ :

Proposition 3: Let $\Delta_{1}, \Delta_{2}$ be the discriminants of $f_{1}(\lambda):=$ $\operatorname{det}(\lambda A-B)=0, f_{2}(\lambda):=\operatorname{det}(\lambda B-A)=0$, respectively, where $A, B \in \mathbb{R}^{3 \times 3}$. Then $\Delta_{1}=\Delta_{2}$.

Proof: Let $\operatorname{det}(\lambda A-B)=0 \Leftrightarrow f_{1}(\lambda):=c_{3} \lambda^{3}+$ $c_{2} \lambda^{2}+c_{1} \lambda+c_{0}=0$, with $c_{\ell} \in \mathbb{R}, \forall \ell \in\{0, \ldots, 3\}$. It can be verified that $\operatorname{det}(\lambda B-A)=0 \Leftrightarrow f_{2}(\lambda)=$ $-c_{0} \lambda^{3}-c_{1} \lambda^{2}-c_{2} \lambda-c_{3}=0$. Let $\lambda_{1}, \lambda_{2}, \lambda_{3}$ be the solutions of $f_{1}(\lambda)=0$, i.e. $f_{1}\left(\lambda_{1}\right)=f_{1}\left(\lambda_{2}\right)=f_{1}\left(\lambda_{3}\right)=0$, and $\lambda_{1} \lambda_{2} \lambda_{3}=-\frac{c_{0}}{c_{3}}$. By substituting $\frac{1}{\lambda_{\ell}}$ in $f_{2}(\lambda), \ell \in\{1,2,3\}$, we obtain $-c_{0} \lambda_{\ell}^{-3}-c_{1} \lambda_{\ell}^{-2}-c_{2} \lambda_{\ell}^{-1}-c_{3}=-\left(c_{3} \lambda_{\ell}^{3}+\right.$ $\left.c_{2} \lambda_{\ell}^{2}+c_{1} \lambda_{\ell}+c_{0}\right)=-f_{1}\left(\lambda_{\ell}\right)=0$. Hence, $\frac{1}{\lambda_{1}}, \frac{1}{\lambda_{2}}, \frac{1}{\lambda_{3}}$ are the solutions of $f_{2}(\lambda)=0$. The discriminants of $f_{1}(\lambda)=0$ and $f_{2}(\lambda)=0$ are $\Delta_{1}=c_{3}^{4}\left(\lambda_{1}-\lambda_{2}\right)^{2}\left(\lambda_{1}-\lambda_{3}\right)^{2}\left(\lambda_{2}-\lambda_{3}\right)^{2}$ and $\Delta_{2}=\left(-c_{0}\right)^{4}\left(\lambda_{1}^{-1}-\lambda_{2}^{-1}\right)^{2}\left(\lambda_{1}^{-1}-\lambda_{3}^{-1}\right)^{2}\left(\lambda_{2}^{-1}-\lambda_{3}^{-1}\right)^{2}=$ $c_{0}^{4}\left(\lambda_{1} \lambda_{2} \lambda_{3}\right)^{-4}\left(\lambda_{2}-\lambda_{1}\right)^{2}\left(\lambda_{3}-\lambda_{1}\right)^{2}\left(\lambda_{3}-\lambda_{2}\right)^{2}$, which, by substituting $c_{0}=-c_{3} \lambda_{1} \lambda_{2} \lambda_{3}$, becomes $\Delta_{2}=\Delta_{1}$.

Therefore, we conclude that the discriminants $\Delta_{i, j}^{s}\left(x_{i}, x_{j}\right)$ and $\Delta_{j, i}^{s}\left(x_{j}, x_{i}\right)$ of $\operatorname{det}\left(\lambda A_{i}^{s}\left(x_{i}\right)-A_{j}^{s}\left(x_{j}\right)\right)=0$ and $\operatorname{det}\left(\lambda A_{j}^{s}\left(x_{j}\right)-A_{i}^{s}\left(x_{i}\right)\right)=0$, respectively, are the same, for all $s \in\{x y, x z, y z\}$. Hence, we can define uniquely for each edge $m \in \overline{\mathcal{M}}$ the continuously differentiable function $\Delta_{m}: \mathbb{M}^{2} \rightarrow \mathbb{R}_{\geq 0}$, with

$$
\begin{aligned}
& \Delta_{m}\left(x_{m_{1}}, x_{m_{2}}\right):=\sigma\left(\Delta_{m_{1}, m_{2}}^{x y}\left(x_{m_{1}}, x_{m_{2}}\right)\right)+ \\
& \quad \sigma\left(\Delta_{m_{1}, m_{2}}^{x z}\left(x_{m_{1}}, x_{m_{2}}\right)\right)+\sigma\left(\Delta_{m_{1}, m_{2}}^{y z}\left(x_{m_{1}}, x_{m_{2}}\right)\right),
\end{aligned}
$$

which needs to remain positive for all times in order to achieve the collision avoidance property, i.e., $\Delta_{m}\left(x_{m_{1}}(t), x_{m_{2}}(t)\right)>$ $0, \forall t \in \mathbb{R}_{\geq 0}, m \in \overline{\mathcal{M}}$. Note that, in view of Proposition 3, the agents $m_{1}$ and $m_{2}$ can calculate (3) based on $\Delta_{m_{1}, m_{2}}^{s}\left(x_{m_{1}}, x_{m_{2}}\right)$ and $\Delta_{m_{2}, m_{1}}^{s}\left(x_{m_{2}}, x_{m_{1}}\right)$, respectively, $\forall s \in\{x y, x z, y z\}, m \in \overline{\mathcal{M}}$.

We still need to incorporate the fact the that agents have a limited sensing radius, and that agent $i$ does not have access to the functions $\Delta_{i, j}^{s}\left(x_{i}, x_{j}\right)$, when $j \notin \mathcal{N}_{i}(p)$. To that end, we define first the greatest lower bound of $\Delta_{m}$ when both agents $m_{1}, m_{2}$ are in each other's sensing radius, i.e., $\widetilde{\Delta}_{m}:=\inf _{\left(x_{m_{1}}, x_{m_{2}}\right) \in \mathbb{M}^{2}}\left\{\Delta_{m}\left(x_{m_{1}}, x_{m_{2}}\right)\right.$ s.t. $\| p_{m_{1}}-$ $\left.p_{m_{2}} \| \leq \min \left\{d_{\operatorname{con}_{m_{1}}}, d_{\text {con }_{m_{2}}}\right\}\right\}, \forall m \in \overline{\mathcal{M}}$. Since $d_{\mathrm{con}, i}>$ $\max \left\{l_{x, i}, l_{y, i}, l_{z, i}\right\}+\max _{j \in \mathcal{N}}\left\{\max \left\{l_{x, j}, l_{y, j}, l_{z, j}\right\}\right\}+\varepsilon, \forall i \in$ $\mathcal{N}$, it follows that there exists a positive constant $\varepsilon_{\Delta}$ such that $\widetilde{\Delta}_{m} \geq \varepsilon_{\Delta}>0, \forall m \in \overline{\mathcal{M}}$. Next, we define the smooth switching functions $\beta_{m}: \mathbb{R}_{\geq 0} \rightarrow\left[0, \bar{\beta}_{m}\right]$, with [9]

$$
\beta_{m}(x)=\bar{\beta}_{m} \frac{\sigma(x)}{\sigma(x)+\sigma\left(\bar{\Delta}_{m}-x\right)},
$$

where $\bar{\Delta}_{m}$ is a positive constant satisfying $\bar{\Delta}_{m}<\widetilde{\Delta}_{m}, \forall m \in$ $\overline{\mathcal{M}}$. Then, by choosing $\beta_{m}:=\beta_{m}\left(\gamma_{\sigma} \Delta_{m}\left(x_{m_{1}}, x_{m_{2}}\right)\right)$, where $\gamma_{\sigma}$ is a positive scaling constant, we incorporate the limited sensing radius of the agents in the collision avoidance scheme, since $\frac{\partial \beta_{m}(x)}{\partial x}$ vanishes when $m_{1} \notin \mathcal{N}_{m_{2}}(p)$ or $m_{2} \notin \mathcal{N}_{m_{1}}(p)$, i.e., when at least one of the agents that form edge $m$ lies outside the sensing range of the other agent. The terms $\bar{\beta}_{m}$ can be any positive constants, $\forall m \in \mathcal{M}$. All the necessary information for the construction of the functions $\beta_{m}, \Delta_{m}$, i.e., the constants $\bar{\Delta}_{m}, \bar{\beta}_{m}$ and the lengths $l_{x, i}, l_{y, i}, l_{z, i}, i \in \mathcal{N}$, can be transmitted off-line to the agents.

We can now define a suitable barrier function for each edge $m \in \overline{\mathcal{M}}$ as any continuously differentiable function $b_{m}: \mathbb{R}_{\geq 0} \rightarrow \mathbb{R}_{\geq 0}$ with the property $\lim _{x \rightarrow 0} b_{m}(x)=\infty$, e.g., $b_{m}(x)=\frac{1}{x}, m \in \overline{\mathcal{M}}$. The barrier function for edge $m$ is then $b_{m}:=b_{m}\left(\beta_{m}\right), \forall m \in \overline{\mathcal{M}}$.

We propose now a decentralized feedback control law for the solution of Problem 1. Firstly, we define the estimations of the unknown terms $\theta_{i} \in \mathbb{R}^{\ell}$ and $d_{b_{i}} \in \mathbb{R}$ of Property 2 and Assumption 1, respectively, as $\hat{\theta}_{i} \in \mathbb{R}^{\ell}$ and $\hat{d}_{b_{i}} \in \mathbb{R}$, with the respective errors $\widetilde{\theta}_{i}:=\hat{\theta}_{i}-\theta_{i}$ and $\widetilde{d}_{b_{i}}:=\hat{d}_{b_{i}}-d_{b_{i}}, \forall i \in \mathcal{N}$. By using adaptive and discontinuous control techniques, we prove in the following that these estimations compensate appropriately for the unknown terms, without necessarily converging to them. In particular, we design the feedback control laws for $u_{s, i}$ as

$$
\begin{aligned}
u_{s, i}= & \sum_{m \in \overline{\mathcal{M}}} \alpha(i, m) \kappa_{m} \bar{E}_{\eta}\left(\eta_{i}\right)^{\top} \frac{\partial \Delta_{m}}{\partial x_{i}}+Y_{i}\left(x_{i}\right) \hat{\theta}_{i} \\
& -k_{v_{i}} v_{i}-\hat{d}_{b_{i}} \operatorname{sgn}\left(v_{i}\right),
\end{aligned}
$$

where $\alpha(i, m)=-1$ if agent $i$ is part of edge $m$, and $\alpha(i, m)=0$ otherwise, $\forall i \in \mathcal{N}, m \in \overline{\mathcal{M}}$, and $\kappa_{m}:=$ $\frac{\partial b_{m}\left(\beta_{m}\right)}{\partial \beta_{m}} \frac{\partial \beta_{m}\left(\Delta_{m}\right)}{\partial \Delta_{m}}, \forall m \in \overline{\mathcal{M}}$. Moreover, we design the associated adaptation laws

$$
\left.\begin{array}{ccc}
\dot{\hat{\theta}}_{i} & := & -\gamma_{i, \theta} Y_{i}\left(x_{i}\right)^{\top} v_{i} \\
\dot{\hat{d}}_{b_{i}} & := & \gamma_{i, d}\left\|v_{i}\right\|_{1}
\end{array}\right\} \forall i \in \mathcal{N},
$$

with arbitrary bounded initial conditions, where $\gamma_{\theta, i}$ and $\gamma_{d, i}$ are positive gains, $\forall i \in \mathcal{N}$. The correctness of (5)-(6) is shown in the following theorem:

Theorem 2: Consider a multi-agent system comprised of 3D ellipsoidal agents and subject to the dynamics (2) at a collision-free initial configuration, i.e., $\mathcal{A}_{i}\left(x_{i}(0)\right) \cap$ $\mathcal{A}_{j}\left(x_{j}(0)\right)=\emptyset, \forall i, j \in \mathcal{N}$ with $i \neq j$. Then, application of the control and adaptation laws (5), (6) guarantees that the agents avoid collisions for all times, i.e., $\mathcal{A}_{i}\left(x_{i}(t)\right) \cap \mathcal{A}_{j}\left(x_{j}(t)\right)=\emptyset$, 
$\forall i, j \in \mathcal{N}$ with $i \neq j, t \in \mathbb{R}_{>0}$, with all closed loop signals being bounded. Moreover, $\lim _{t \rightarrow \infty} v_{i}(t)=0, \forall i \in \mathcal{N}$.

Proof: Consider the vector $\zeta_{x}:=\left[x^{\top}, v^{\top}, \widetilde{\theta}^{\top}, \widetilde{d}_{b}^{\top}\right]^{\top}$ $\in \mathcal{Z}_{x}:=\mathcal{X} \times \mathbb{R}^{7 N+\ell N}$, where $\mathcal{X}:=\left\{x \in \mathbb{M}^{N}: \mathcal{A}_{i}\left(x_{i}\right) \cap\right.$ $\left.\mathcal{A}_{j}\left(x_{j}\right)=\emptyset, \forall i, j \in \mathcal{N}, i \neq j\right\}$ as defined in Section III, $\underset{\widetilde{\theta}}{v}:=\left[v_{1}^{\top}, \ldots, v_{\widetilde{\theta}}^{\top}\right]^{\top} \in \mathbb{R}^{6 N}, \widetilde{d}_{b}:=\left[\widetilde{d}_{b_{1}}, \ldots, \widetilde{d}_{b_{N}}\right]^{\top} \in \mathbb{R}^{N}$, $\widetilde{\theta}:=\left[\widetilde{\theta}_{1}^{\top}, \ldots, \widetilde{\theta}_{N}^{\top}\right] \in \mathbb{R}^{\ell N}$. Since the initial configuration is collision-free, it holds that $\zeta_{x}(0) \in \mathcal{Z}_{x}$. By combining (2), (5), and (6), we obtain the closed-loop system dynamics $\dot{\zeta}_{x}=$ $F_{\zeta}\left(\zeta_{x}, t\right)$. It can be verified that $F_{\zeta}$ is measurable in $t$ over $\mathbb{R}_{\geq 0}$ and measurable and locally bounded in $\zeta_{x}$ over $\mathcal{Z}_{x}$. Hence, by invoking Prop. 3 of [27], we conclude that at least one Filippov solution exists and any such solution satisfies $\zeta_{x}:\left[0, t_{1}\right) \rightarrow \mathcal{Z}_{\underline{x}}$ for a positive $t_{1}$. Define $\zeta:=\left[\phi, b_{1}, \ldots, b_{\bar{M}}, v^{\top}, \widetilde{\theta}^{\top}, \widetilde{d}_{b}^{\top}\right]^{\top}$ $\in \mathcal{Z}:=\mathbb{R}^{\bar{M}+7 N+\ell N+1}$, where $\phi$ is the cooperative term defined in Section III. Note that $\zeta(0) \in \mathcal{Z}$ and, for any finite $r, \zeta \in \overline{\mathcal{B}}(0, r) \subset \mathcal{Z} \Leftrightarrow \zeta_{x} \in \mathcal{Z}_{x}$, which we prove in the following. Define the function $V(\zeta):=\phi(x)+\sum_{m \in \overline{\mathcal{M}}} b_{m}+$ $\sum_{i \in \mathcal{N}}\left\{\frac{1}{2} v_{i}^{\top} M_{i}\left(x_{i}\right) v_{i}+\frac{1}{2 \gamma_{i, d}} \widetilde{d}_{b_{i}}^{2}+\frac{1}{2 \gamma_{i, \theta}}\left\|\widetilde{\theta}_{i}\right\|^{2}\right\}$, for which it holds that $W_{1}(\zeta) \leq V(\zeta) \leq W_{2}(\zeta)$ for positive definite functions $W_{1}, W_{2}$ on $\mathcal{Z}$. Since $\zeta(0) \in \mathcal{Z}$, we conclude that $V(\zeta(0))$ is well defined, and hence there exists a finite constant $\bar{V}$ such $V(\zeta(0)) \leq \bar{V}$ and $b_{m}(0) \leq \bar{V}, \forall m \in \overline{\mathcal{M}}$. We aim to show that $V$, given its initial boundedness, remains bounded $\forall t \in \mathbb{R}_{>0}$, and so do the terms $b_{m}, \forall m \in \overline{\mathcal{M}}$. By differentiating $\bar{V}$ along the solutions of the closed loop system and in view of Lemma 1 we obtain $\dot{V} \in \dot{\tilde{V}}:=\cap_{\xi \in \partial V(\zeta)} \xi^{\top} \mathrm{K}[\dot{\zeta}]$. Since $V$ is continuously differentiable, the generalized gradient reduces to the standard gradient and therefore, after using Properties 1, 2, and grouping terms, we obtain

$$
\begin{aligned}
& \max _{z \in \tilde{\tilde{V}}}\{z\} \leq \sum_{i \in \mathcal{N}}\left\{\sum_{m \in \mathcal{M}}\left[\alpha(i, m) \kappa_{m} \frac{\partial \Delta_{m}}{\partial x_{i}} \bar{E}_{\eta}\left(\eta_{i}\right)\right] v_{i}+\right. \\
& \left\|v_{i}\right\|_{1}\left\|d_{i}(t)\right\|_{1}+v_{i}^{\top}\left(u_{i}-Y_{i}\left(x_{i}\right) \theta_{i}+\bar{E}_{\eta}\left(\eta_{i}\right)^{\top} \frac{\partial \phi(x)}{\partial x_{i}}\right) \\
& \left.-v_{i} f_{i}\left(v_{i}\right)+\frac{1}{\gamma_{i, d}} \widetilde{d}_{b_{i}} \dot{\hat{d}}_{b_{i}}+\frac{1}{\gamma_{i, \theta}} \widetilde{\theta}_{i}^{\top} \dot{\hat{\theta}}_{i}\right\},
\end{aligned}
$$

By also using Property 3, Assumption 1, substituting $u_{i}=$ $u_{f, i}+u_{s, i}$ with $u_{f, i}=\bar{E}_{\eta}\left(\eta_{i}\right)^{\top} \frac{\partial \phi(x)}{\partial x_{i}}$ and (5), the adaptation laws (6), and using $\widetilde{d}_{b_{i}}=\hat{d}_{b_{i}}-d_{b_{i}}, \widetilde{\theta}_{i}=\hat{\theta}_{i}-\theta_{i}$ and the property $x^{\top} \operatorname{sign}(x)=\|x\|_{1}, \forall x \in \mathbb{R}^{n}$, we obtain $\max _{z \in \dot{\tilde{V}}}\{z\} \leq-\sum_{i \in \mathcal{N}} k_{v_{i}}\left\|v_{i}\right\|^{2}=: W(\zeta)$. Therefore, $z \leq$ $-W(\zeta(t)), \forall z \in \dot{\tilde{V}}(\zeta(t)), t \in\left[0, t_{1}\right)$, where $W: \mathcal{Z} \rightarrow \mathbb{R}_{\geq 0}$ is a positive semi-definite function defined on $\mathcal{Z}$. Hence, by applying Theorem 1, we conclude that $t_{1}=\infty, \zeta(t)$ is bounded in the compact set $\left\{\zeta \in \overline{\mathcal{B}}(0, r): W_{2}(\zeta) \leq c\right\}, \forall t \in \mathbb{R}_{\geq 0}$ for any $r$ and $c$ satisfying $\overline{\mathcal{B}}(0, r) \subset \mathcal{Z}, c<\min _{\|x\|=r} W_{1}(\bar{\zeta})$, and $\lim _{t \rightarrow \infty} W(\zeta(t))=0 \Rightarrow \lim _{t \rightarrow \infty} v(t)=0$. Note that, since the sets $\left\{x \in \mathbb{M}^{N}: x=\phi^{-1}(y), x \in \mathcal{X}\right\}$ are nonempty, $r$ can be chosen arbitrarily large, corresponding to all collision-free initial configurations. Therefore, interagent collisions are avoided, and the adaptation signals $\hat{\theta}_{i}$, $\hat{d}_{b_{i}}$, remain bounded, $\forall i \in \mathcal{N}, t \in \mathbb{R}_{>0}$. The continuity of the terms $Y_{i}(\cdot)$ implies also their boundedness and hence the boundedness of the control signals (5), (6), $t \in \mathbb{R}_{\geq 0}$. Note that convergence of $\widetilde{\theta}_{i}, \widetilde{d}_{b_{i}}$ to zero is not needed, and hence we do not require persistence of excitation or sufficient richness of the respective signals, as is usually assumed in adaptive control when parameter convergence is taken into account.

Remark 1: It can be verified that $\operatorname{det}\left(\lambda A_{m_{1}}^{s}\left(x_{m_{1}}\right)-\right.$ $A_{m_{2}}^{s}\left(x_{m_{2}}\right)$, and hence $b_{m}$, are functions of $p_{m_{1}}-p_{m_{2}}, \eta_{m_{1}}$, $\eta_{m_{2}}$. Therefore, if $\phi$ is a function of $\widetilde{x}$, the aforementioned analysis still holds by setting $\mathcal{X}=\left\{\widetilde{x} \in \mathbb{R}^{\frac{3 N(N-1)}{2}} \times S^{3}\right.$ : $\left.\mathcal{A}_{i}\left(x_{i}\right) \cap \mathcal{A}_{j}\left(x_{j}\right)=\emptyset, \forall i, j \in \mathcal{N}, i \neq j\right\}$. Moreover, note that achievement of the objectives expressed by $\phi$ is not pursued in this paper and may not be necessarily guaranteed due to the potentially counteracting terms of $u_{i}$. The control scheme could be extended, however, by appropriately designing the terms $\phi(x)$ such that the resulting configuration of the agents implies $\phi(x)=0$, like, e.g., [3], [4], [9].

Remark 2: Since $\Delta_{i, j}^{s}=\Delta_{j, i}^{s}$ (due to Proposition 3), $\forall i, j \in \mathcal{N}, i \neq j$, the control scheme can be extended to directed communication graphs, by setting for the $i$ th agent $b_{i, j}=b_{i, j}\left(\beta_{i_{j}}\left(\Delta_{i, j}\left(x_{i}, x_{j}\right)\right)\right), \forall j \in \mathcal{N} \backslash\{i\}$, with $\Delta_{i, j}\left(x_{i}, x_{j}\right)$ as in (3) and $\beta_{i_{j}}$ as in (4), $\widetilde{\Delta}_{i, j}:=$ $\inf _{\left(x_{i}, x_{j}\right) \in \mathbb{M}^{2}}\left\{\Delta_{i, j}\left(x_{i}, x_{j}\right)\right.$ s.t. $\left.\left\|p_{i}-p_{j}\right\| \leq d_{\text {con }_{i}}\right\}$, and appropriately modifying the control law. Similarly, collision avoidance with static environment obstacles could be incorporated in the overall scheme. An event-triggered extension is also possible by employing results from the related literature, e.g., [28]. Finally, for implementation purposes in real applications, the discontinuous part of (5) could be approximated by a continuous function via the boundary layer technique [29], e.g., $\frac{\left|v_{i_{j}}\right|}{\left|v_{i_{j}}\right|+\epsilon_{i_{j}}}$ instead of $\operatorname{sgn}\left(v_{i_{j}}\right)$, when $\left|v_{i_{j}}\right|<\epsilon_{i_{j}}$, where $v_{i_{j}} \in \mathbb{R}$ is the $j$ th component of $v_{i}, \forall i \in \mathcal{N}$, and $\epsilon_{i j}$ is a small positive constant.

\section{SIMULATION RESULTS}

We consider a simulation example with $N=8$ rigid bodies in $\mathrm{SE}(3)$, described by ellipsoids with axes lengths $l_{x, i}=0.5 \mathrm{~m}, l_{y, i}=0.3 \mathrm{~m}, l_{z, i}=0.2 \mathrm{~m}, \forall i \in \mathcal{N}$. The initial poses are $p_{1}=[3,3,0]^{\top}, p_{2}=-[3,3,0]^{\top}, p_{3}=[3,-3,0]^{\top}, p_{4}$ $=[-3,3,0]^{\top}, p_{5}=[3,3,3]^{\top}, p_{6}=-[3,3,3]^{\top}, p_{7}=[3,-3,3]^{\top}$, $p_{8}=[-3,3,-3]^{\top}, \eta_{1}=\eta_{8}=[0.769,0.1696,0.6153,0.0358]^{\top}$, $\eta_{2}=\eta_{6}=[0.8488,-0.3913,-0.0598,-0.3505]^{\top}, \quad \eta_{3}=$ $\eta_{5}=[0.7638,-0.5283,-0.3275,-0.1738]^{\top}, \quad \eta_{4}=\eta_{7}=$ $[0.7257,0.3081,0.3714,0.4904]^{\top}$. We consider that $\phi(x)$ describes an independent multi-agent navigation objective, with desired configurations as $p_{1_{\mathrm{d}}}=p_{2}, p_{2_{\mathrm{d}}}=p_{1}, p_{3_{\mathrm{d}}}=p_{4}$, $p_{4_{\mathrm{d}}}=p_{3}, p_{5_{\mathrm{d}}}=p_{6}, p_{6_{\mathrm{d}}}=p_{5}, p_{7_{\mathrm{d}}}=p_{8}, p_{8_{\mathrm{d}}}=p_{7}, \eta_{i_{\mathrm{d}}}=$ $[1,0,0,0]^{\top}, \forall i \in \mathcal{N}$. We set the errors $e_{p_{i}}:=p_{i}-p_{i_{\mathrm{d}}}$ and $e_{\eta_{i}}:=\left[e_{\varphi_{i}}, e_{\epsilon_{i}}^{\top}\right]^{\top}:=\eta_{i_{\mathrm{d}}} \otimes \bar{\eta}_{i}$, where $\bar{\eta}_{i}:=\left[\varphi_{i},-\epsilon_{i}^{\top}\right]^{\top} \in \mathrm{S}^{3}$ is the quaternion conjugate, $\otimes$ denotes the quaternion product, and $e_{\varphi_{i}}, e_{\epsilon_{i}}$ are the scalar and vector parts, respectively, of the quaternion error [25]. The desired quaternion configuration is achieved when $e_{\eta_{i}}=[ \pm 1,0,0,0]^{\top}$ and hence the function $\phi(x)$ is chosen as $\phi(x)=\sum_{i \in \mathcal{N}}\left(\frac{1}{2}\left\|p_{i}-p_{i_{\mathrm{d}}}\right\|^{2}+1-e_{\varphi_{i}}^{2}\right)$, with $\dot{\phi}=\sum_{i \in \mathcal{N}}\left(\left(p_{i}-p_{i_{\mathrm{d}}}\right)^{\top} \dot{p}_{i}-e_{\varphi_{i}} e_{\epsilon_{i}}^{\top} \omega_{i}\right)$ [25]. The control inputs $u_{f, i}$ are therefore chosen as $u_{f, i}=\left[p_{i_{\mathrm{d}}}^{\top}-\right.$ $\left.p_{i}^{\top}, e_{\varphi_{i}} e_{\epsilon_{i}}^{\top}\right]^{\top}, \forall i \in \mathcal{N}$. The agent masses are chosen as $(0.1,0.2,0.01,0.1,0.1,0.2,0.1,0.2)$ and the principal moments of inertia as $\operatorname{diag}\{0.05,0.03,0.01\}, \forall i \in \mathcal{N}$. We also set 
$f_{i}\left(v_{i}\right)=m_{f_{i}} \sin \left(w_{f_{i}} t+\phi_{f_{i}}\right) v_{i}, d_{i}(t)=\left(1 / m_{f_{i}}\right) \sin \left(w_{f_{i}} t+\right.$ $\left.\phi_{f_{i}}\right), \forall i \in \mathcal{N}$, with $\left[m_{f_{1}}, \ldots, m_{f_{8}}\right]=0.1 \cdot[1,2,0.1,1,1,2,1,2]$, $\left[\omega_{f_{1}}, \ldots, \omega_{f_{8}}\right]=0.01 \cdot[1,2,0.1,1,1,2,1,2]$, and $\left[\phi_{f_{1}}, \ldots, \phi_{f_{8}}\right]$ $=0.01 \cdot[5,1,0.05,0.5,1,0.5,1]$. We choose $b_{m}=\frac{1}{\beta_{m}}$, with $\bar{\beta}_{m}=1, \bar{\Delta}_{m}=10^{4}, \gamma_{\sigma}=10^{-40}, \forall m \in \mathcal{M}$, and $\hat{\theta}_{i}(0)=0.1, \hat{d}_{b_{i}}(0)=0.2, k_{v_{i}}=1, \forall i \in \mathcal{N}$. The expressions for $\Delta_{m}\left(x_{m_{1}}, x_{m_{2}}\right)$ were derived by using the symbolic toolbox of MATLAB. Fig. 2 shows a 3D plot of the agent trajectories, and Fig. 3 shows the minimum of the barrier functions $\min _{m \in \mathcal{M}}\left\{b_{m}(t)\right\}$ (left), which is always positive, and the signals $\gamma_{i}(t):=\left\|p_{i}-p_{i_{\mathrm{d}}}\right\|^{2}+1-e_{\varphi_{i}}^{2}$ and $v_{i}(t)$ (right), $\forall i \in \mathcal{N}, t \in[0,15]$. A short video that demonstrates the aforementioned simulation example can be found in https://youtu.be/IAni7zIMM7k.

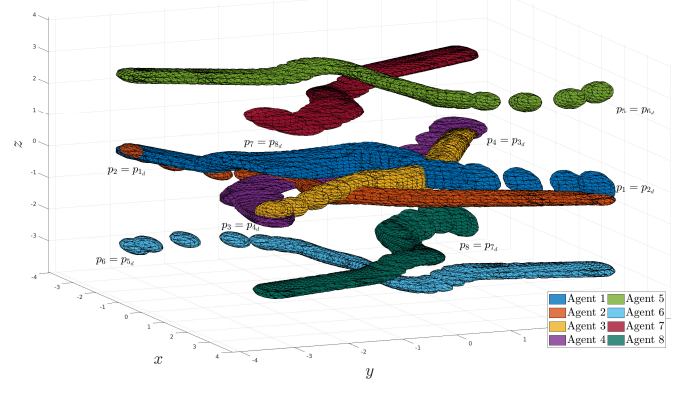

Fig. 2. The evolution of agent trajectories $\forall t \in[0,15]$.
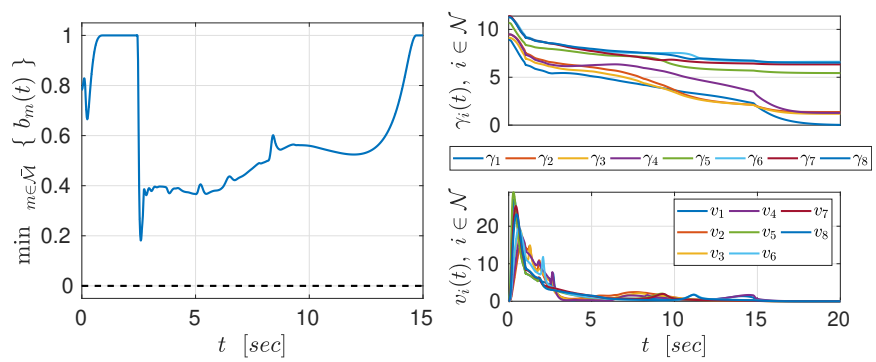

Fig. 3. Left: The evolution of the minimum of the barrier functions $\min _{m \in \mathcal{M}}\left\{b_{m}(t)\right\}$. Right: The evolution of the signals $\gamma_{i}(t)$ and $v_{i}(t)$, $\forall i \in \mathcal{N}, \forall t \in[0,15]$.

\section{CONCLUSIONS AND FUTURE WORK}

This paper proposes a closed-form barrier function as well as a robust decentralized control scheme for the multi-agent collision avoidance of $3 \mathrm{D}$ ellipsoids, using discontinuous and adaptive controllers. Future efforts will be devoted towards adding connectivity properties to the current framework and resolving issues of local minima.

\section{REFERENCES}

[1] E. Rimon and D. E. Koditschek, "Exact robot navigation using artificial potential functions," IEEE Transactions on robotics and automation, vol. 8, no. 5, pp. 501-518, 1992.

[2] C. Vrohidis, P. Vlantis, C. P. Bechlioulis, and K. J. Kyriakopoulos, "Prescribed time scale robot navigation," IEEE Robotics and Automation Letters, vol. 3, no. 2, pp. 1191-1198, 2018.
[3] D. Panagou, D. M. Stipanović, and P. G. Voulgaris, "Distributed coordination control for multi-robot networks using lyapunov-like barrier functions," IEEE Transactions on Automatic Control, vol. 61, no. 3, pp. 617-632, 2015.

[4] D. V. Dimarogonas, S. G. Loizou, K. J. Kyriakopoulos, and M. M. Zavlanos, "A feedback stabilization and collision avoidance scheme for multiple independent non-point agents," Automatica, vol. 42, no. 2, pp. 229-243, 2006.

[5] S. G. Loizou, "The multi-agent navigation transformation: Tuning-free multi-robot navigation.," in Robotics: Science and Systems, vol. 6 , pp. 1516-1523, 2014

[6] C. K. Verginis, Z. Xu, and D. V. Dimarogonas, "Decentralized motion planning with collision avoidance for a team of uavs under high level goals," IEEE Intern. Conf. Rob. and Autom., pp. 781-787, 2017.

[7] C. K. Verginis, A. Nikou, and D. V. Dimarogonas, "Robust formation control in se (3) for tree-graph structures with prescribed transient and steady state performance," Automatica, vol. 103, pp. 538-548, 2019.

[8] L. Sabattini, C. Secchi, and N. Chopra, "Decentralized connectivity maintenance for networked lagrangian dynamical systems with collision avoidance," Asian Journal of Control, vol. 17, no. 1, 2015.

[9] S. G. Loizou, "The navigation transformation," IEEE Transactions on Robotics, vol. 33, no. 6, pp. 1516-1523, 2017.

[10] P. Vlantis, C. Vrohidis, C. P. Bechlioulis, and K. J. Kyriakopoulos, "Robot navigation in complex workspaces using harmonic maps," IEEE International Conference on Robotics and Automation, 2018.

[11] H. G. Tanner, S. G. Loizou, and K. J. Kyriakopoulos, "Nonholonomic navigation and control of cooperating mobile manipulators," IEEE Transactions on robotics and automation, vol. 19, no. 1, 2003.

[12] C. K. Verginis and D. V. Dimarogonas, "Multi-agent motion planning and object transportation under high level goals," IFAC-PapersOnLine, vol. 50, no. 1, pp. 15816-15821, 2017.

[13] Y. Yan and G. S. Chirikjian, "Closed-form characterization of the minkowski sum and difference of two ellipsoids," Geometriae Dedicata, vol. 177, no. 1, pp. 103-128, 2015.

[14] E. Rimon and S. P. Boyd, "Obstacle collision detection using best ellipsoid fit," Journ. Intell. Rob. Syst., vol. 18, no. 2, pp. 105-126, 1997.

[15] A. Best, S. Narang, and D. Manocha, "Real-time reciprocal collision avoidance with elliptical agents," IEEE International Conference on Robotics and Automation, pp. 298-305, 2016.

[16] C. K. Verginis, A. Nikou, and D. V. Dimarogonas, "Communicationbased decentralized cooperative object transportation using nonlinear model predictive control," in 2018 European Control Conference (ECC), pp. 733-738, 2018.

[17] M. Z. Romdlony and B. Jayawardhana, "Stabilization with guaranteed safety using control lyapunov-barrier function," Automatica, 2016.

[18] X. Xu, "Constrained control of input-output linearizable systems using control sharing barrier functions," Automatica, 2018.

[19] L. Wang, A. D. Ames, and M. Egerstedt, "Safety barrier certificates for collisions-free multirobot systems," IEEE Transactions on Robotics, vol. 33, no. 3, pp. 661-674, 2017.

[20] L. Lindemann and D. V. Dimarogonas, "Control barrier functions for signal temporal logic tasks," IEEE control systems letters, vol. 3, no. 1, pp. 96-101, 2019 .

[21] C. K. Verginis and D. V. Dimarogonas, "Robust decentralized abstractions for multiple mobile manipulators," IEEE Conference on Decision and Control, pp. 2222-2227, 2017.

[22] B. Paden and S. Sastry, "A calculus for computing filippov's differential inclusion with application to the variable structure control of robot manipulators," IEEE transactions on circuits and systems, 1987.

[23] Y.-K. Choi, W. Wang, L. Yang, and M.-S. Kim, "Continuous collision detection for two moving elliptic disks," IEEE Transactions on Robotics, vol. 22, no. 2, 2006.

[24] N. Fischer, R. Kamalapurkar, and W. E. Dixon, "Lasalle-yoshizawa corollaries for nonsmooth systems," IEEE Transactions on Automatic Control, vol. 58, no. 9, pp. 2333-2338, 2013.

[25] C. K. Verginis, M. Mastellaro, and D. V. Dimarogonas, "Robust cooperative manipulation without force/torque measurements: Control design and experiments," IEEE Trans. Contr. Syst. Technol., 2019.

[26] R. Kelly, V. S. Davila, and J. A. L. Perez, Control of robot manipulators in joint space. Springer Science \& Business Media, 2006.

[27] J. Cortes, "Discontinuous dynamical systems," IEEE control Systems, vol. 28 , no. 3, 2008.

[28] P. Tabuada, "Event-triggered real-time scheduling of stabilizing control tasks," IEEE Transactions on Automatic Control, vol. 52, no. 9, 2007.

[29] I. M. Boiko, "Chattering in sliding mode control systems with boundary layer approximation of discontinuous control," International Journal of Systems Science, vol. 44, no. 6, pp. 1126-1133, 2013. 